\title{
«Sterben in Raten» des Spitals Wattwil?
}

\author{
D. Güntert
}

Ich schreibe diesen Artikel als Arzt in der freien Praxis und besorgter Bürger, weil mir die Entwicklung im Gesundheitswesen generell und speziell in unserer Region Toggenburg Sorge bereitet. In einer ersten Runde wurden bereits vor sechs Jahren diverse Regionalspitäler in mehreren Kantonen geschlossen. Zentralisierung in Richtung Agglomerationszentren ist das «Geheimrezept» der Ökonomen, wodurch die nötigen Einsparungen gemacht werden sollen. Obwohl unzählige Beispiele das Gegenteil beweisen und belegen, dass Zentrumsspitäler die einzelnen Patienten durchschnittlich bedeutend teurer behandeln, wird dennoch an dieser Strategie festgehalten. Das gebetsmühlenartige Wiederholen dieser falschen Argumente macht die Lügen aber nicht wahrer. Sie bleiben aber in den Köpfen der Bürger hängen und verwischen die Tatsachen. Wahr hingegen ist, dass seit der KVG-Revision Bund und Kantone sich massgeblich aus der Beteiligung der Gesundheitskosten zurückgezogen haben. Diese nun fehlenden Subventionen mussten nach «unten» umverteilt werden, d.h. an Leistungserbringer und Patienten. In einer nächsten Runde werden nun die Landregionen sukzessive ihrer Infrastrukturen beraubt und Agglomerationszentren angegliedert.

\section{Ereignisse der letzten sechs Jahre}

Vor sechs Jahren waren im Rahmen der Gesundheitsreformen drastische Sparmassnahmen eingeleitet worden. Diese veranlassten Schliessungen diverser Regionalspitäler. Vorreiterin war die Gesundheitsdirektion (GD) Zürich mit Vorsteherin, Regierungsrätin Verena Diener. Damals wurden die Massnahmen unter dem Schlagwort Rationalisierung in die Wege geleitet. Die GD Zürich betonte gebetsmühlenartig, dass die Qualität der Grundversorgung keinesfalls herabgesetzt werden soll bzw. dessen Erhaltung prioritäres Ziel ist. Es gehe nicht um Rationierung, sondern um Rationalisierung. Neun Spitäler schlossen im Kanton Zürich mit der neuerstellten Spitalliste '98 ihren Betrieb. Letztes Jahr erklärte RR Verena Diener, dass die bisherigen Bemühungen unzureichend waren und dass nun doch Rationierungsmassnahmen nötig seien. Dabei sei tatsächlich mit Qualitätseinbussen in der Grund- versorgung zu rechnen (Sanierungsprogramm SAN 04, Juni 2004). In einer Informationsveranstaltung für das Kader im Gesundheitswesen der GD St. Gallen vom 24. Juni 2004 wurde von Ökonomen Bilanz gezogen und festgehalten, dass all die bisher durchgeführten Bemühungen keine effektiven Einsparungen zur Folge hatten. Sogar von Ökonomen wurde also konstatiert, dass die bislang verfolgten Strategien ineffizient waren; dennoch wird von GD und Bund weiterhin an derselben Sparschraube gedreht.

\section{Regierung und GD veranlassen neue Verwaltungen}

Im Rahmen der Spitalliste '98 standen auch Regionalspitäler im Toggenburg zur Diskussion; so die Spitäler Wil, Flawil und Wattwil. Damals standen die Zeichen für das Spital Wil auf Messers Schneide. Alle Spitäler wurden schliesslich in die Liste aufgenommen. Dieser Entschluss hatte zur Folge, dass nötige Sanierungsmassnahmen über mehrere Millionen Franken investiert wurden. Bevölkerung und Spitäler selbst wähnten sich in Sicherheit. Sechs Jahre später standen alle drei Spitäler wieder zur Diskussion. Im Auftrag der Regierung wurde ein Verwaltungsratsgremium eingesetzt, das ausschliesslich Vertreter ausserhalb der Region Toggenburg beinhaltete. Dieses Gremium, der Verwaltungsrat der Spitalregion Fürstenland-Toggenburg (Spitalregion 4), kam erklärtermassen vorwiegend aus betriebswirtschaftlichen Gründen zum Schluss, dass die Spitäler Wattwil und Flawil geschlossen werden müssen und dass eine Zentralisierung auf die Region Wil zu erfolgen habe. Die Argumentation im Schlussbericht lässt allerdings auch unter rein ökonomischen Aspekten viele Fragen offen und hinterlässt grosse Zweifel am tatsächlichen Spareffekt, insbesondere, als Zahlen über die daraus folgenden Investitionen zum Ausbau des Spitals Wil gänzlich fehlten.

\section{Heftige Reaktionen aus der Bevölkerung}

Dieser Verwaltungsratsbeschluss hatte wohl eher wider Erwarten heftige Protestreaktionen bei der Toggenburger Bevölkerung hervorgerufen. 
Demonstrationen und unzählige Leserbriefe zeigten, dass die Bürger keineswegs mit dem Entscheid einverstanden waren. Informationsveranstaltungen mit dem damaligen Gesundheitsvorsteher, Regierungsrat Anton Grüninger, vermochten die Bedenken nicht auszuräumen. Kurz darauf musste RR Grüninger sein Amt verlassen. Die Nachfolgerin, Regierungsrätin Verena Hanselmann, revidierte den Entscheid des Verwaltungsrats. Die GD St. Gallen entschied, dass das Spital als Akutspital erhalten bleiben solle, dass aber die Abteilung für Gynäkologie und Geburtshilfe geschlossen und nach Wil verlegt werden soll. Dieser Beschluss löste anfänglich grosse Erleichterung bei der Bevölkerung aus. Es zeigte sich aber im weiteren Verlauf, dass dieser Entscheid folgenschwerer ausfiel, als er anfänglich den Anschein machte. Ärzteverein und besorgte Stimmen aus der Bevölkerung machten auf das Problem einer damit eingeschränkten Grundversorgung aufmerksam und befürchteten einen schleichenden Abbau der Spitalleistungen und damit ein stetes Aushöhlen des Spitals, was schliesslich einem Sterben in Raten gleichkäme. Wenige Wochen nach Veröffentlichung des Regierungsratsentscheids löste die Chefärztin für Anästhesie ihr Arbeitsverhältnis auf. Fehlende Unterstützung und fehlende Einbezugnahme in solch schwerwiegende Entscheidungen von seiten der Geschäftsführung wurden angeführt. Kurze Zeit später kündigten ein engagierter Oberarzt der Chirurgie, die Oberhebamme der Geburtshilfe und schliesslich der Chefarzt für Gynäkologie kurzfristig.

\section{Übernahme der Spitalführung durch den Kanton}

Seit Übernahme der Regionalspitäler Wattwil, Flawil, Wil und Uznach durch den Kanton durch die sogenannte Quadriga vor zwei Jahren sind einschneidende Umstrukturierungen in den Spitalführungen vorgenommen worden, die hinter verschlossener Tür entschieden wurden. Nachdem das Spital Wattwil durch eine solide, wirtschaftlich und medizinisch vernünftige Führung über Jahrzehnte einen guten Ruf in der Bevölkerung verbuchen konnte, steht nach dessen Übernahme durch den Kanton innert zweier Jahre dessen Existenz auf dem Spiel. GD und Geschäftsleitung beteuern zwar unentwegt, dass das Spital Wattwil als Akutspital erhalten bleiben soll. Tatsache aber ist, dass bisherige Leistungen verschwinden (Gynäkologe und Geburtshilfe), dass Fusionen auf Chefarztebene zugunsten des Spitals Wil vorgenommen werden (nach Kündi- gung der Chefärztin für Anästhesie in Wattwil wird neuer Leiter beider Spitäler der Anästhesiechefarzt von Wil). Die Erweiterung und der Ausbau des Spitals Wil sind schon lange in vollem Gange, während in Wattwil Umstrukturierungen vorgenommen werden und Kaderpersonal verschwindet. Diese Bedenken, die auch vom regionalen Ärzteverein deutlich zum Ausdruck gebracht wurden, fanden bei der Geschäftsleitung kein Gehör.

\section{Regionalplan wird umgesetzt}

Schon lange sind schweizweite grundlegende oder, moderner ausgedrückt, nachhaltige Reformen im Gange, deren Ursprung aus Vordenkerorganisationen (sogenannter Think-Tanks) stammen. Diese geben richtungsweisende Anleitungen, wie die Gesellschaft in der Zukunft auszusehen hat. Eine solche Vordenkerorganisation der Wirtschaft ist die Avenir Suisse (vgl. «Der Toggenburger» vom 15. Februar 2005, S. 10). Deren Studie «fordert Grossregionen als neue politische Ebene zwischen Bund und Kantonen». Es sollen anstelle der Kantone sogenannte Zweckregionen geschaffen werden. Diese sollen «kantonsübergreifende Aufgaben wie Verkehr, Sicherheit, Gesundheits- und Bildungswesen» übernehmen. Sechs Grossregionen oder Metropolitanregionen (Zürich, Basel, Bern, Tessin, Lausanne und Genf) sowie sechs regionale Agglomerationen (St. Gallen, Sion, Neuenburg, Olten, Luzern und Lugano) ersetzen dabei die bisherigen 26 Kantone und 3000 Gemeinden. Handelt es sich tatsächlich «nur» um theoretische Phantasten, die Gedankenspiele betreiben, oder sind hier doch nachhaltige Reformen im Gange, die wir zuwenig wahrnehmen oder zuwenig ernst nehmen? Die Grossregionen sollen sogar über die Landesgrenzen hinausreichen. Diese theoretischen Pläne findet man in Europa schon längst umgesetzt. Im Kanton Schaffhausen sollen nach Anleitung eines «Jahrhundertreformprojekts» die bisherigen 32 Gemeinden zu sieben Grossgemeinden fusioniert werden, und dies auch unter Zwang, wenn nötig («Der Toggenburger», 21. Februar 2005, S. 7).

\section{Zentralisierung und Regionalplan}

Das obenerwähnte weitergedacht, würde bedeuten, dass im Gesundheitswesen entsprechend den Grossregionen zentralisiert werden müsste. Das wiederum lässt folgern, dass periphere Spitäler, Regionalspitäler und Bezirksspitäler in 
ihrer Funktion überflüssig werden, so dass sie entweder geschlossen, fusioniert oder zu anderen Zwecken umfunktioniert werden. Ich glaube, dass die eingangs erwähnten Schilderungen passende Beispiele dafür sind. Zweifellos gehört das Spital Wattwil zu einer Randregion. Der Kanton St. Gallen gehört nicht einmal zu den Grossregionen, sondern zu einer regionalen Agglomeration. Der Entscheid des Verwaltungsrats der Spitalregion 4 vom Dezember letzten Jahres, der nota bene im Auftrag und Einverständnis der Regierung gehandelt hat, hat die Schliessung von zwei Regionalspitälern entschieden (Flawil, Wattwil). Dieser Entscheid wurde wie bereits beschrieben nur wegen heftiger Gegenwehr verhindert (oder nur aufgeschoben?). Die Region Wil und vor allem die Region St. Gallen werden zunehmend ausgebaut. Es ist hier wohl kaum zu übersehen, dass Zentralisierungsbestrebungen im Gange sind, die ein längerfristiges Ziel verfolgen.

\section{Rolle von Bund und Kanton}

Wer spielt aber in diesem Spiel die zentrale Rolle? Woher kommen die Devisen und Anleitungen? Wer ist Zahnrädchen in einem grösseren Getriebe, wer ist Zahnrädchenschleifer, wer ist am Steuerknüppel und wer lässt sich einfach in einem Boot mittreiben, von dem er nicht weiss, wohin es fährt? Ohne Zweifel ist die Rolle des Bundes eine unrühmliche, weil der Bund gegen den Willen der Bevölkerung und entgegen demokratischem Recht grundlegende Änderungen vornimmt. Reformen werden nach Salamitaktik durchgeführt, ohne den Bürger nur einmal zu fragen, ob er damit auch einverstanden ist. Gebetsmühlenartig wiederholte Argumente wie die Schere zwischen Leistungen und Finanzierungspotential klaffe immer weiter auseinander, wir könnten uns unsere Luxusmedizin nicht mehr leisten usw., ohne dass glaubhafte Belege vorgelegt werden, machen die Lügen nicht wahrer. Der Bund legt seine tatsächlichen Absichten nicht klar auf den Tisch, nämlich einen steten Abbau der Grundversorgung mit Rationierungsmassnahmen auf mittelmässiges EU-Niveau. Dies tut er weder im Gesundheits-, noch im Bildungswesen, noch bei der schleichenden Integration in die EU, genausowenig wie in unzähligen anderen Belangen auch. Der Bevölkerung wird plumpe Wahlpropaganda an den Kopf geworfen, wie man sie aus den USA und aus den Oststaaten kennt. Hier ist klar eine offene, sachliche und transparente Diskussion und Information vonnöten. Nur so können wir ernsthaft und nachhaltig die vorhandenen Probleme im Gesundheitswesen lösen.

\section{Was lernen wir daraus?}

Reformen werden nicht ohne längerfristiges Ziel in die Wege geleitet. Reformen sind Resultat von langjährigen Vorbereitungen auf verschiedenen Ebenen, die ein Ziel verfolgen. Das erstrebte Ziel, nicht die kleinen Zwischenschritte, ist ein wesentlicher Aspekt, um Reformen verstehen und erkennen zu können. Das Ziel muss aber einerseits allen Beteiligten inkl. Bürgern bekannt sein und muss andererseits von den Reformern offen dargelegt werden. Beide Partien sind entscheidend in diesem Prozess beteiligt. Der eine Teil sind diejenigen, die das Steuer in der Hand halten und das Boot lenken; der andere Teil sind diejenigen, die im Boot sitzen. Diejenigen, die im Boot sitzen müssen, aber eingreifen, wenn das Boot in die falsche Richtung gesteuert wird und auf den Wasserfall zusteuert.

\section{Aufwachen aus dem Dornröschenschlaf}

Das Schicksal aller Regionalspitäler ist in weiterer Zukunft ungewiss. Die Qualität und Kontinuität in der Grundversorgung der Randregionen ist meiner Ansicht nach ernsthaft in Gefahr. Keineswegs ungewiss ist aber, dass von ökonomischer und politischer Seite stetig Massnahmen in die Wege geleitet werden, die Agglomerationszentren stärken und Landregionen ausbluten lassen, da sie wirtschaftlich ein Hemmschuh sind. Die Berg- und Randregionen und damit auch die Spitäler sollen von den rentierenden städtischen Agglomerationen abgekoppelt werden. Wir Ärzte müssen ernsthaft aus unserem Dornröschenschlaf aufwachen und über unsere Nasenspitze hinausschauen. Denn hier sind nachhaltige Umstrukturierungen im Gange, die nicht mehr so rasch rückgängig gemacht werden können. In diesem Prozess haben wir ein Wörtchen mitzureden. Wir müssen es aber auch tun. 